\title{
Calcium coordination in aqueous solution from atomistic simulations
}

\author{
HUGO MOISON ${ }^{1}$, MERLIN MÉHEUT ${ }^{2}$ AND MAGALI \\ BENOIT $^{3}$
}

${ }^{1}$ CNRS GET

${ }^{2}$ CNRS Géosciences Environment Toulouse, Université de Toulouse, UPS, IRD, CNES

${ }^{3}$ CEMES, CNRS

Presenting Author: hugo.moison@get.omp.eu

The isotope fractionation properties of dissolved calcium at thermodynamical equilibrium are subject to debate. These properties depend on coordination number, lower coordinations being enriched in heavy isotopes ([6]). Calcium in aqueous solution has been studied experimentally (e.g. [4]) with coordination numbers varying from 5.1 to 10 . DFT based ab initio molecular dynamics (AIMD) may clarify parameters governing the coordination at the atomic level. Despite a large number of studies (e.g. [1]), there is still no consensus on $\mathrm{Ca}$ coordination, with numbers ranging from 5.9 to 7.0. Technical and physical arguments can be invoked. Technically, uncertainties remain on the time necessary to equilibrate, and on the correct functional to choose. Physically, Ca coordination may be influenced by concentration or temperature. Another important, yet ill-evaluated parameter, is the mean residence time (MRT). This one intervenes in the dynamic of the calcium and so in kinetic isotope fractionation ([3]). In this work, we performed Car-Parrinello AIMD of $\mathrm{Ca}^{2+}(\mathrm{aq})$, with and without counter-ions $(\mathrm{Cl}-)$ at $300 \mathrm{~K}$ and $400 \mathrm{~K}$. We used the BLYP, PBE and PBEsol functionals, in relation with a parallel study on solids ([5]). Theoretical frameworks accounting for Van der Waals interactions (BLYP with Grimme-D2 correction, VdW-DF2 functional) were also considered as they best describe pure water ([2]). To assess equilibration, trajectories were carried out up to $120 \mathrm{ps}$. For BLYP, BLYP with Grimme-D2 and VdW-DF2, equilibration was achieved, for the rest, it is still unclear. We will discuss the relationship between the time necessary for equilibration, the MRT, and water structure, in relation with existing experimental data on pure water structure. Similarly with previous studies, we found that $\left[\mathrm{Ca}(\mathrm{H} 2 \mathrm{O})_{6}\right]^{2+}$ and $\left[\mathrm{Ca}(\mathrm{H} 2 \mathrm{O})_{7}\right]^{2+}$ complexes are the most likely to form, with average coordination varying from 5.98 to 7.05 depending on functional. Trajectories with dispersion terms tend to increase this number. The influence of concentration will also be discussed.

[1] Baer and Mundy. (2016) JPCB 120, 1885-1893

[2] Gillan et al. (2016) JCP 144, 130901

[3] Hofmann et al. (2012) PNAS 109, 18689-18694

[4] Megyes et al. (2004) JPCB 108, 7261-7271

[5] Méheut (2021) Goldschmidt poster

[6] Moynier and Fujii (2017) Sci Rep 7, 44255. 